\title{
Normal glycosylation screening does not rule out SRD5A3-CDG
}

European Journal of Human Genetics (2011) 19, 1019; doi:10.1038/ejhg.2010.260; published online 13 July 2011

By applying next-generation sequencing in three affected siblings showing mental retardation, cataract, coloboma, dysmorphic features and kyphosis, Kahrizi et al. ${ }^{1}$ reported a novel mutation in the recently discovered $5 \alpha$-reductase type 3 (SRD5A3) gene. ${ }^{2}$ The authors suggest that Kahrizi syndrome (MIM 612713) is allelic to the novel type of congenital disorder of glycosylation (SRD5A3-CDG), presenting with variable degree of cerebellar abnormalities, ataxia and eye malformations and caused by different SRD5A3 mutations. ${ }^{2,3}$ They emphasize the need for direct genetic testing for SRD5A3 in patients with coloboma or cataract in combination with mental retardation. However, the potential for biochemical screening of glycosylation abnormalities was underestimated on the basis of insufficient information.

Screening for CDG in the index family (CVH385) clearly showed abnormal $\mathrm{N}$-glycosylation of transferrin, as described by Cantagrel et al. ${ }^{2}$ In addition, the CDG screening of the original patient described by Al-Gazali et al. ${ }^{4}$ was only reported to be normal initially, but was clearly abnormal in three different samples at different ages (6 months, 1 year and 10 years) using radioimmuneassay, transferrin isoelectric focusing (TIEF) or mass spectrometry. ${ }^{2}$ Furthermore, all patients, as described by Cantagrel et al. ${ }^{2}$ and by Morava et al. ${ }^{3}$ show abnormal CDG type I glycosylation profiles on multiple occasions.

We also would like to emphasize that the biochemical mechanism, underlying the somewhat clinically similar Peters' Plus syndrome (MIM 261540), is completely different. The abnormal Glc $\beta 1-3 F u c$ O-linked disaccharides on thrombospondin type 1 repeats of Peters' Plus syndrome ${ }^{5}$ are not present in serum transferrin and, thus, TIEF screening is not indicated in the diagnostics of this disease.

It is important to note that so far all other patients reported with SRD5A3 mutations were children, and Kahrizi's paper ${ }^{1}$ is the first report on adults. Abnormal glycosylation evaluated in plasma might be less evident with aging, and TIEF might become normal, especially in adults. ${ }^{6}$ These variable and age-dependent changes in serum glycosylation pattern (eg, normal results at old or very young age) are known for other types of CDG as well. ${ }^{7}$ Owing to the indicative cerebello-ocular presentation in SRD5A3-CDG, we agree with the authors' remark to continue with direct testing for SRD5A3 mutations in highly suspected cases, even when CDG screening is normal.

\section{CONFLICT OF INTEREST}

The authors declare no conflict of interest.

Miski Mohamed ${ }^{1,2}$, Vincent Cantagrel ${ }^{3}$, Lihadh Al-Gazali ${ }^{4}$, Ron A Wevers ${ }^{1,5}$, Dirk J Lefeber ${ }^{1,5,6}$ and Eva Morava ${ }^{1,2}$ ${ }^{1}$ Institute for Genetic and Metabolic Disease, Radboud University Nijmegen Medical Centre, Nijmegen, The Netherlands;

${ }^{2}$ Department of Pediatrics, Radboud University Nijmegen Medical Centre, Nijmegen, The Netherlands;

${ }^{3}$ Department of Neurosciences and Pediatrics, Howard Hughes Medical Institute, University of California, San Diego, CA, USA;

${ }^{4}$ Departments of Pediatrics and Pathology, Faculty of Medicine and Health Sciences, United Arab Emirates University, Al Ain, UAE; ${ }^{5}$ Department of Laboratory Medicine, Laboratory for Genetic, Endocrine and Metabolic Disease, Radboud University Nijmegen Medical Centre,

Nijmegen, The Netherlands;

${ }^{6}$ Department of Neurology, Radboud University Nijmegen Medical Centre, Nijmegen, The Netherlands E-mail:E.Morava@cukz.umcn.nl

1 Kahrizi K, Hu CH, Garshasbi M et al: Next generation sequencing in a family with autosomal recessive Kahrizi syndrome (OMIM 612713) reveals a homozygous frameshift mutation in SRD5A3. Eur J Hum Genet 2011; 19: 115-117.

2 Cantagrel V, Lefeber DJ, Ng BG et al: SRD5A3 is required for converting polyprenol to dolichol and is mutated in a congenital glycosylation disorder. Cell 2010; 142 . 203-217.

3 Morava E, Wevers RA, Cantagrel V et al: A novel cerebello-ocular syndrome with abnormal glycosylation due to abnormalities in dolichol metabolism. Brain 2010; 133: 3210-3220

4 Al-Gazali L, Hertecant J, Algawi K, EI TH, Dattani M: A new autosomal recessive syndrome of ocular colobomas, ichthyosis, brain malformations and endocrine abnormalities in an inbred Emirati family. Am J Med Genet A 2008; 146: 813-819.

5 Hess D, Keusch JJ, Oberstein SA, Hennekam RC, Hofsteenge J: Peters Plus syndrome is a new congenital disorder of glycosylation and involves defective Omicron-glycosylation of thrombospondin type 1 repeats. J Biol Chem 2008; 283: 7354-7360.

6 Vermeer S, Kremer HP, Leijten QH et al: Cerebellar ataxia and congenital disorder of glycosylation la (CDG-la) with normal routine CDG screening. J Neurol 2007; 254: 1356-1358.

7 Morava E, Guillard M, Lefeber DJ, Wevers RA: Autosomal recessive cutis laxa syndrome revisited. Eur J Hum Genet 2009; 17: 1099-1110. 